\title{
Flow of Oil and Water through Elastic Polymer Gels
}

\author{
H.H. Al-Sharii' ${ }^{1}$, C.A. Grattoni' ${ }^{1}$, R.A. Dawe ${ }^{2}$ and R.W. Zimmerman' \\ 1 T.H. Huxley School of Environment, Earth Sciences and Engineering, Imperial College of Science, Technology and Medicine, \\ London SW7 2BP - United Kingdom \\ 2 Department of Chemical Engineering, University of West Indies, St. Augustine - Trinidad \\ e-mail: radawe@tstt.net.tt - r.zimmerman@ic.ac.uk
}

\begin{abstract}
Résumé - Écoulement de pétrole et d'eau à travers des gels de polymère élastiques - La production massive d'eau associée à l'exploitation des puits compte parmi les problèmes majeurs que connaît l'industrie pétrolière. Une des méthodes de contrôle de la production d'eau à avoir été utilisée avec succès est l'injection de gels de polymère aux abords du puits. Malheureusement, l'injection de gels de polymère n'est pas toujours synonyme de réussite. Ceci s'explique en partie par une connaissance incomplète des mécanismes réduisant la perméabilité de l'eau plus fortement que celle du pétrole (réduction disproportionnée de la perméabilité).
\end{abstract}

Nous avons réalisé une série d'expérimentations sur l'écoulement d'eau et de pétrole à travers un volume de gel de polymère et un micromodèle rempli de gels de polymère, afin de comprendre les mécanismes impliqués dans la réduction disproportionnée de la perméabilité. Des écoulements expérimentaux à travers des polyacrylamides gels faibles ont été effectués dans le but d'obtenir les perméabilités du gel à des conditions différentes. Les perméabilités respectives du pétrole et de l'eau à travers le gel de polymère s'avèrent dépendantes du débit suivant une fonction puissance, mais avec des pré-facteurs et des exposants différents.

Les expérimentations d'écoulement à l'échelle microscopique ont été menées dans des modèles de verre transparent afin de visualiser distinctivement l'écoulement. Nos observations nous ont permis d'éliminer plusieurs explications proposées auparavant et d'identifier le fait que le pétrole et l'eau peuvent se déplacer à travers les mêmes canaux, mais de manière différente, plus particulièrement à l'échelle des pores. L'eau s'écoule à travers le gel de polymère comme si elle s'écoulait de manière diffusive dans le milieu poreux, alors que le pétrole trouve un chemin sous forme de gouttes immiscibles ou filaments. Cette différence de régime d'écoulement donne lieu à une réduction disproportionnée de la perméabilité.

Mots-clés : gels de polymère, écoulement diphasique, milieu poreux, micromodèle, réduction de la perméabilité.

Abstract - Flow of Oil and Water through Elastic Polymer Gels - High water production is one of the major problems faced by the petroleum industry. One method of controlling water production is to inject polymer gels into the near-wellbore formation. Unfortunately, polymer gel injections are not always successful, in part because the exact mechanisms by which they reduce water permeability more than oil permeability (i.e., Disproportionate Permeability Reduction, DPR) are not understood.

We have conducted a series of experiments on flow of water and oil through bulk polymer gels and through polymer-filled micromodels to elucidate the fundamental mechanisms involved in DPR. Flow experiments of oil and water through weak polyacrylamide-based gels have been performed to obtain the gel permeabilities under different test conditions. Oil and water permeabilities through the gel were each found to vary with flow rate according to a power-law, but with different pre-factors and exponents. 
The micro-scale flow experiments were conducted in transparent glass models to visualise clearly the flow events. Our observations enabled us to discount many previously-proposed explanations, and identify the fact that the oil and water can travel through the same pore channels, but in ways that differ, particularly at the pore scale. Water flows through the gel matrix as if flowing by diffusive flow through a porous medium, whereas the oil pushes its way in the form of immiscible drops or filaments. This difference in flow regime gives rise to the measured disproportionate permeability reduction.

Keywords: polymer gels, two-phase flow, porous media, micromodels, permeability reduction.

\section{INTRODUCTION}

Many wells in oil or gas reservoirs produce unwanted water in addition to the desired hydrocarbons. The producing water/oil or water/gas ratios tend to increase during the life of a well, so, as more reservoirs become mature, this problem is becoming acute world-wide. Water production is troublesome for three main reasons: the high costs of producing and separating out the water, the difficulties in disposing of it in an environmentally benign manner, and the corrosive effects of the high-salinity water on pipes and other collection equipment. One method that is used to control water production is to inject polymer gels into the porous rocks near the production wells. Research within the petroleum industry has been directed toward the attainment of better understanding of water control by polymer gels in oil field, so as to improve the success rate of future treatments. Polymers and gels have been developed which, when injected into production wells, reduce the formation permeability to water much more than to oil; this phenomenon is known as disproportionate permeability reduction (DPR). In these treatments, often a viscoelastic gel is formed through a reaction between a polymer solution and crosslinker agent creating a material that is capable of modifying the permeability.

Although DPR is well documented, and many successful polymer gel applications have been reported in the technical literature, the origin and basic physical mechanisms of DPR are not well understood. Suggested mechanisms include wall effects [1], segregated pathways [2,3], and gel shrinking and swelling [4]. None of these explanations have been supported by visual observations, because laboratory experiments are usually performed in rock cores, which by their nature are opaque.

This paper aims to shed some light onto DPR by visualising the mechanisms by which water and oil flow through polymer gels emplaced in transparent glass micromodels. The results of this study are currently being used to provide the physical description and the basic data needed to develop and validate mathematical models representing the oil and water flow through elastic polymer gels [5, 6].

\section{EXPERIMENTAL METHODS AND PROCEDURE}

Flow experiments have been conducted in three sets of experimental models, namely bulk, single capillary pores and transparent two-dimensional porous micromodels with "random" microstructures. Glass capillary pores allow us to conduct the tests at the pore scale, while avoiding the geometric complexity of "real" porous media. Glass micromodels, on the other hand, represent more closely the pore structure of oil-bearing sandstones, although they are quasitwo-dimensional. These models have different pore sizes (from $1 \mathrm{~mm}$ down to $60 \mu \mathrm{m}$ ), thereby allowing the gel and its interaction with the fluids and pore walls to be studied over a range of scales.

The gelant used was polyacrylamide-chromium acetate. Gel permeabilities to water and oil were measured as functions of flow rate, under various test conditions.

\subsection{Materials}

- Water: A saline water solution $2 \% \mathrm{NaCl}$ w/w was used to prepare the gel. The same brine was used for the flow experiments. This solution had a viscosity of $1.0 \times 10^{-3} \mathrm{~Pa} \cdot \mathrm{s}$, and a density of $1014 \mathrm{~kg} / \mathrm{m}^{3}$.

- Oil: Paraffin had a viscosity of $1.7 \times 10^{-3} \mathrm{~Pa} \cdot \mathrm{s}$ and a density of $780 \mathrm{~kg} / \mathrm{m}^{3}$.

- Gel: The polymer used in this study was a high molecular-weight polyacrylamide, Alcoflood 254S polymer (Ciba Specialty Chemicals), which is available in powder form. The polymer has a molecular weight of 500000 and an anionic content of less than 5\%. A solution of $1.8 \%$ (by weight) of polyacrylamide was prepared by slow addition of the polymer powder to brine in a vortex created by magnetic stirring. After the complete dissolution of the polymer, $200 \mathrm{ppm}$ of chromium acetate (crosslinker) was added while stirring. The gelation began after approximately $8 \mathrm{~h}$ at $65^{\circ} \mathrm{C}$, and ceased after about $15 \mathrm{~h}$. The gel has a final strength code $\mathrm{G}$, according to the classification scheme of Sydansk [7].

- Models: The flow behaviour through polymer gel was studied in capillary pores, and porous micromodels. They have different sizes (from $1 \mathrm{~mm}$ down to $60 \mu \mathrm{m}$ ), thereby allowing the gel and its interaction with the pore walls to be studied at different scales.

The capillary pores (square cross-section of 1-mm wide and $7.2-\mathrm{cm}$ length) are built by using three layers of glass plates. The middle layer has two pieces with a gap between them (the pore), and is sintered to the other two layers. The micromodels are produced by etching a pattern into a glass 
plate, after which a flat plate is sintered on top of it. The pore size of the network varies from 60 to $250 \mu \mathrm{m}$, and the total pore volume is approximately $70 \mu \mathrm{l}$. Full details of the fabrication of the micromodels and their applications can be found elsewhere [8].

\subsection{Methods}

The preparation and sequence of flooding used for the micromodel and single pore were as follows:

- evacuate the model, saturate it with $2 \%$ w/w NaCl solution;

- inject two pore volumes of polymer-crosslinker solution at a constant flow rate, then clear all entrances and exits with brine, shut in the model, and put it into a water bath at $65^{\circ} \mathrm{C}$ for $24 \mathrm{~h}$ to allow gelation to occur;

- after gelation, inject dyed brine at various flow rates and pressures, and calculate the permeability of the gel to water;

- inject dyed oil at the same flow rates/pressures as used in the third step, and calculate the permeability of the gel to oil.

A schematic diagram of the experimental set-up is shown in Figure 1. During the flow experiments, the pressure was monitored using a pressure transducer connected to the inlet of the model, and recorded with a computer, while the flow rate was measured at the outlet of the model using a calibrated capillary tube. The permeability was determined by using three different methods: constant inlet pressure, constant flow rate, and the falling head method [9]. The visual observations were obtained through a microscope and camera connected to a video recorder. All experiments were

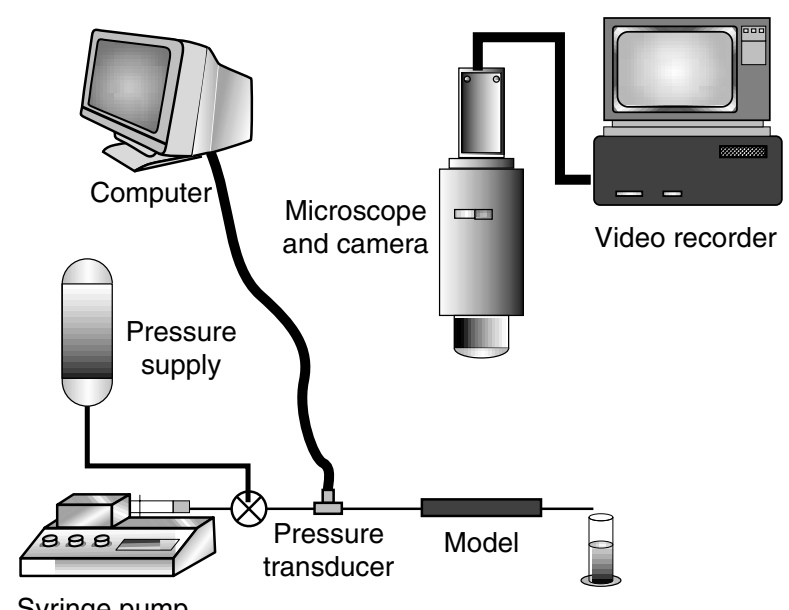

Syringe pump

Figure 1

Experimental set-up for flow tests in micromodels and single capillary pores. performed at room temperature, and the pressure at the outlet of the model was atmospheric.

\section{RESULTS}

\subsection{Gel Permeability to Water and Oil}

The gel permeability was determined by measuring the pressure differentials and flow rates while observing the flow behaviour of water and oil through polymer gels in glass capillary pores and micromodels. The data were interpreted within the context of Darcy's law for flow through porous media [9], which states that:

$$
v=\frac{Q}{A}=\frac{-k \Delta P}{\mu L}
$$

where $Q\left(\mathrm{~m}^{3} / \mathrm{s}\right)$ is the volumetric flux, $A\left(\mathrm{~m}^{2}\right)$ is the crosssectional area, $\Delta P(\mathrm{~Pa})$ is the pressure head differential occurring over length $L(\mathrm{~m})$ of the porous medium, $\mu(\mathrm{Pa} \cdot \mathrm{s})$ is the viscosity of the fluid, and $k\left(\mathrm{~m}^{2}\right)$ is the permeability coefficient of the porous medium. However, the gel permeability is not constant, as can be seen in Figure 2. The permeability is a function of the flow velocity, following a non-linear relationship.

All data sets could be fitted with an effective permeability that varies as a power-law function of the flow velocity:

$$
k_{\mathrm{gel}}=k_{0}\left(\frac{v}{v_{0}}\right)^{n}
$$

where $k_{\text {gel }}\left(\mathrm{m}^{2}\right)$ is the effective permeability of the gel, $k_{0}\left(\mathrm{~m}^{2}\right)$ is a constant pre-factor, $v(\mathrm{~m} / \mathrm{s})$ is the superficial velocity, $n$ is gel "elasticity" index, and $v_{0}(\mathrm{~m} / \mathrm{s})$ is an arbitrary reference velocity. This power-law behaviour held over several orders of magnitude and different boundary conditions (Fig. 2). The power-law was found also to be valid for both water and oil, although with different prefactors and slightly different exponents. In the capillary pore experiments, oil and water were found to have exponents of $n=0.85$ and 0.94 , respectively (Fig. 3), but with significantly different pre-factors of $4.68 \times 10^{-13}$ and $1.74 \times 10^{-15} \mathrm{~m}^{2}$. The effective permeability of the gel to water was thus significantly less than that to oil. In the micromodels, similar values were found for the exponents, but the pre-factors were smaller (Fig. 4). In our experiments, the permeability to oil in a single capillary pore is approximately 200 times larger than the permeability to water, indicating that the DPR effect does not require separate flow paths. In the micromodel, the permeability to oil is approximately four times greater than the permeability to water; the DPR effect is smaller than in capillary pores because the oil drop size is controlled by the small pore sizes (see below). 


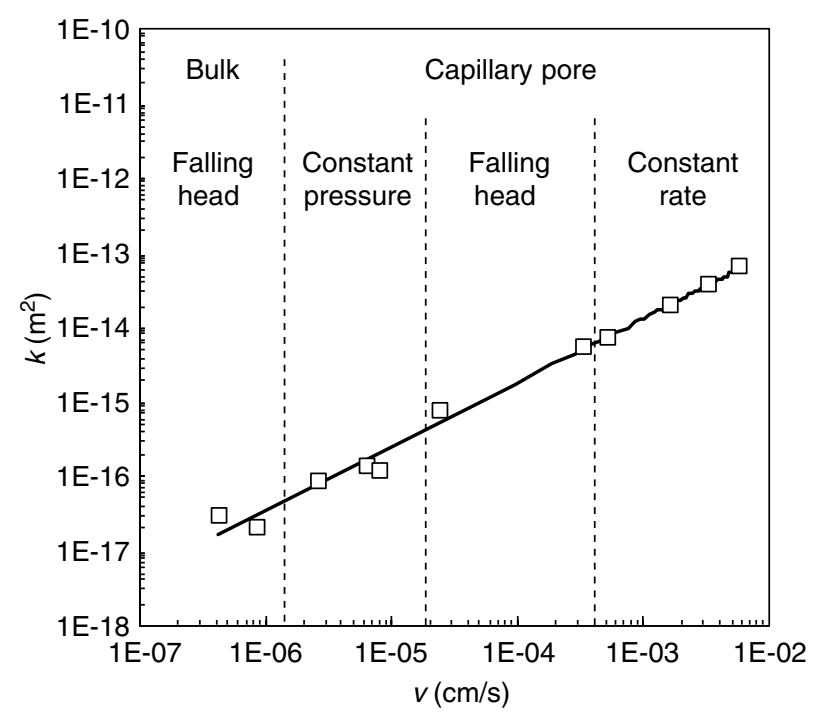

Figure 2

Gel permeability to water as a function of the superficial velocity for different boundary conditions for flow in bulk and in a single capillary pore.

The effective permeability of the gel to oil and water depends on the flow velocity, but appears to be independent of the boundary conditions applied, for the following reason. The bulk gel permeability to water [10] presents a value that fits well with the flow velocity correlation for capillary tubes. This indicates that the effect of the walls of the capillary pore is negligible. It was shown [5] that when the absolute permeability of the capillary is more than 100 times greater than the effective permeability of the gel to water, then the effect of the channel walls is negligible, confirming that the measured effective permeability accurately reflects the properties of the gel.

For a Newtonian fluid in a non-deformable porous medium, the permeability coefficient for the matrix is constant and independent of the fluid, as long as there is no molecular interaction with the pore walls, and the medium is fully saturated with that fluid. However, our experimental results show a non-linear relationship between pressure gradient, $\Delta P / L$, and velocity, $v=Q / A$, implying "non-Darcy" behaviour. Such behaviour is usually observed when a nonNewtonian fluid flows through a rigid porous medium [11]. However, the water and oil used in our studies are both Newtonian fluids, so the non-Darcy behaviour seems to be attributable to the elastic properties of the gel, rather than to the fluids, as discussed below.

The permeability $v s$. flow velocity relationship is parameterised by an "elasticity" index, $n$. If $n$ were equal to zero, the permeability would be constant and not flow-ratedependent. This would be the case if the polymer acted as a

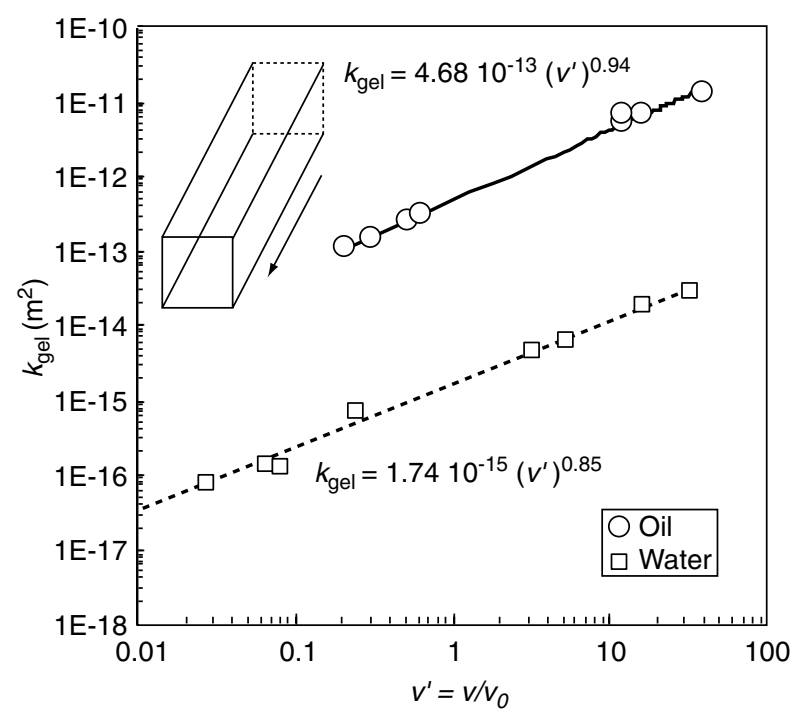

Figure 3

Gel permeability in a single square capillary pore as a function of the dimensionless velocity v' $=v / v_{0}\left(v_{0}=1 \times 10^{-4} \mathrm{~cm} / \mathrm{s}\right)$. The permeability to oil is approximately 200 times greater than the permeability to water.

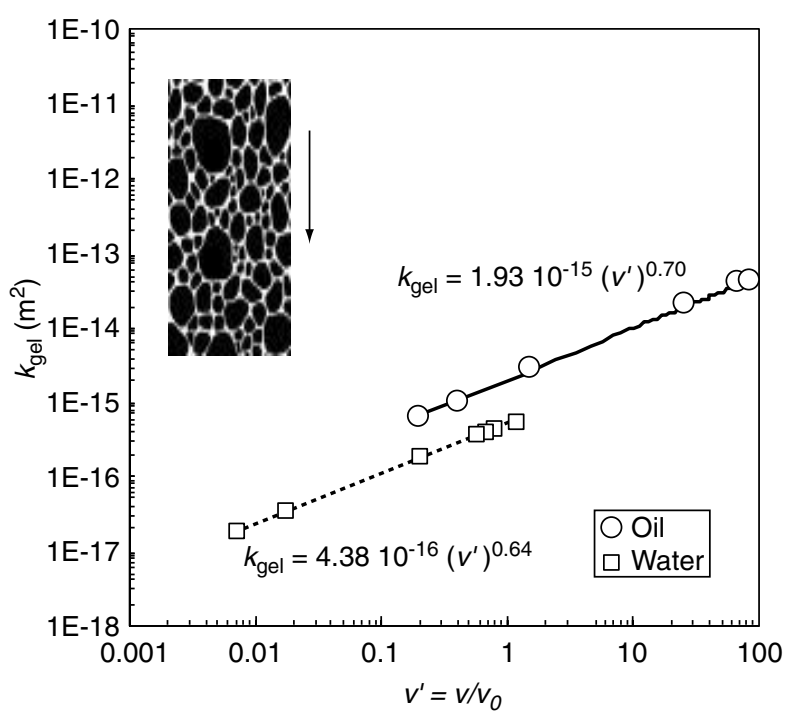

Figure 4

Gel permeability measured in a glass micromodel, as a function of the dimensionless velocity $v^{\prime}=v / v_{0}\left(v_{0}=1 \times 10^{-4} \mathrm{~cm} / \mathrm{s}\right)$. The permeability to oil is approximately four times greater than the permeability to water.

rigid porous medium. As $n$ was found to be greater than 0 , we conclude that the gel is highly deformable (i.e., elastic); attempts are currently under way to develop models to explain this flow velocity dependence in terms of the deformability of the gel. 


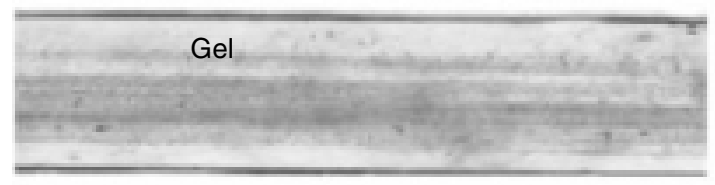

Dyed water flow

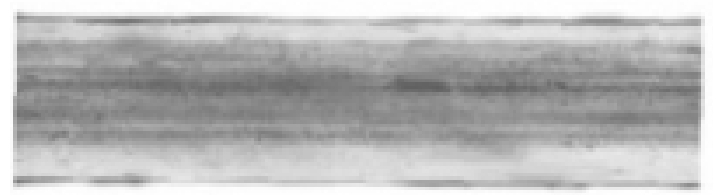

Glass

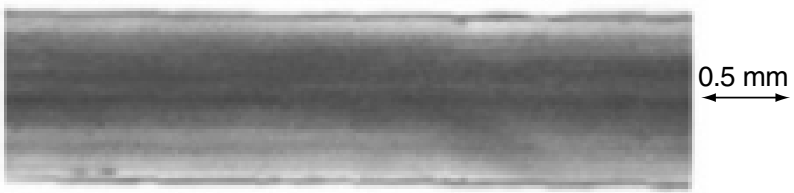

Figure 5

Water flowing through the gel placed in a single capillary pore. The dyed water "diffuses" through the gel matrix, increasing its colour as time increases. The time interval between pictures is $30 \mathrm{~s}$.

\subsection{Flow Visualisation}

In our experiments, the physical processes of the flow of oil and water through the gel emplaced within transparent glass models were observed. These flow processes are both qualitatively and quantitatively different. The most important observation was that water flows through the gel matrix in a "diffusive" manner, as if flowing through a porous medium, whereas the oil pushes its way through the gel in the form of immiscible drops or filaments. As water is the main component (solvent) of the gel, filling the network of crosslinked polymer molecules, and that the injected water is miscible with the water contained within the gel, it will be able to flow through this polymer network [12]. In the channel experiment, during dyed water injection, water was seen to flow through the gel matrix as if flowing through a porous medium, as illustrated in Figure 5. Oil, on the other hand, is immiscible with the water contained within the gel, and is thus unable to flow through the polymer network in this way. The oil must pass through the gel in a manner that minimises the surface area of contact between the oil and the gel. It does so by pushing its way through the gel in the form of immiscible drops or filaments (Figs. 6-9). The oil drops flowing in the single pore are larger than those in the micromodel (Figs. 6 and 7), indicating that the oil drop size is proportional to the pore sizes. Roughly, the diameter of the oil drops or filaments tended to be about one third to one half the diameter of the pore. This range is consistent with the size of fingers observed when a fluid of small viscosity displaces a viscous fluid between two narrowly-spaced plates [13].

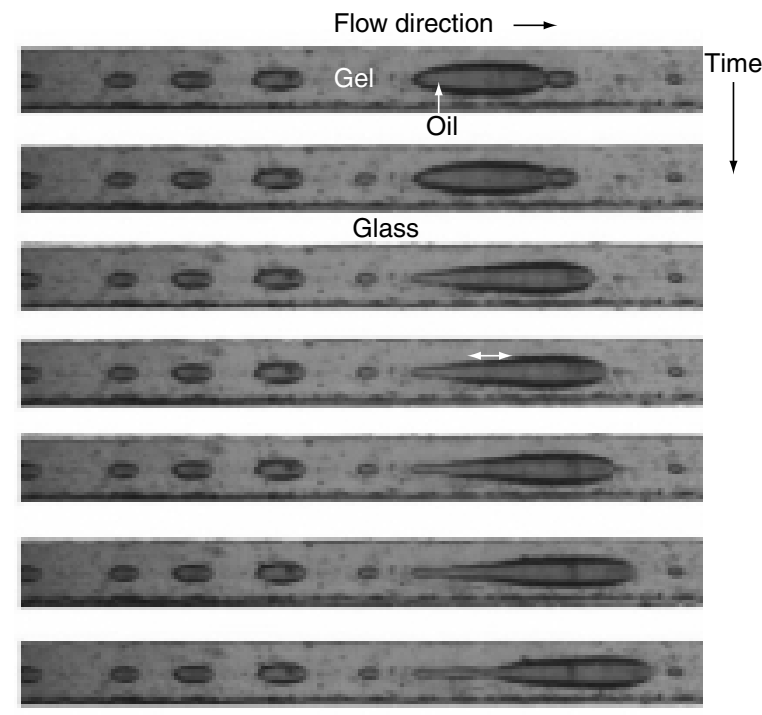

Figure 6

Oil flow through the gel placed in a single capillary pore. The oil pushes through as isolated droplets. The time interval between pictures is $0.01 \mathrm{~s}$.

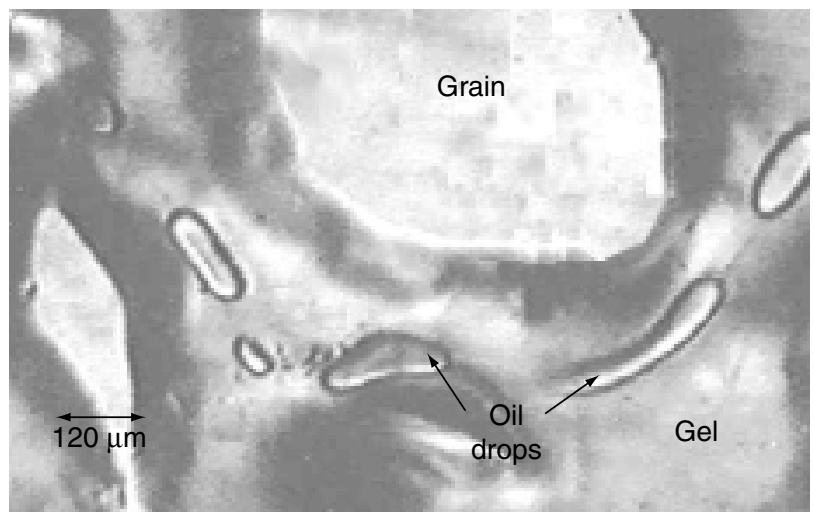

Figure 7

Oil flow through the gel placed in a micromodel. Oil flows as a stream of small drops through the centre of the pore throats.

The oil flows as drops at low flow rates; but above a critical rate these drops can join together to form a continuous filament (Fig. 8). A detailed observation of an oil drop flowing through polymer gel was obtained through a highspeed video camera (recording at a speed of 100 frames/s), shown in Figure 6. As can be observed, the large drop flows through the centre of the cross-section of the channel. This drop coalesces with the small droplets, which are travelling at a lower speed, and at the same time leaves behind it a trail of small droplets. The stream of drops is formed close to the inlet of the channel by snap-off, as can 

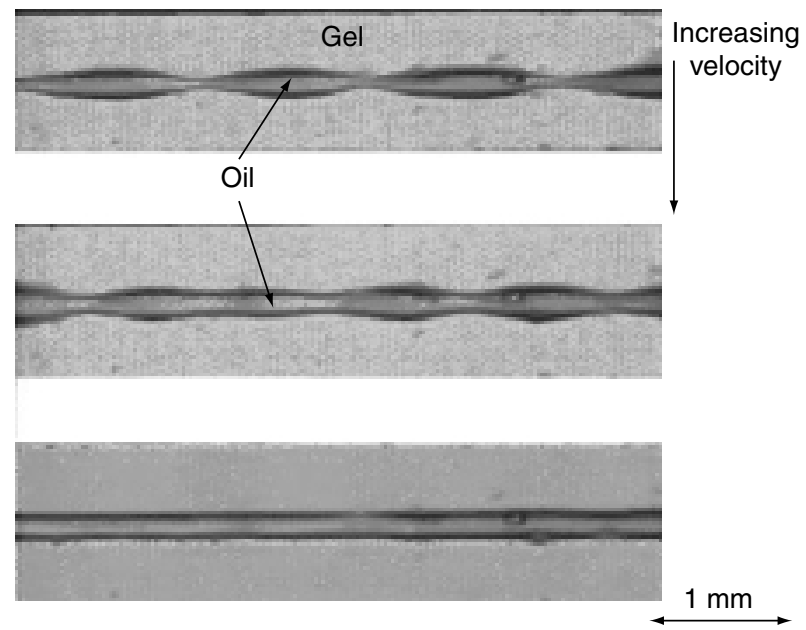

Figure 8

Oil flow through the gel placed in a single capillary pore. The oil drops become more frequent as the velocity increases until a continuous filament of oil is formed.

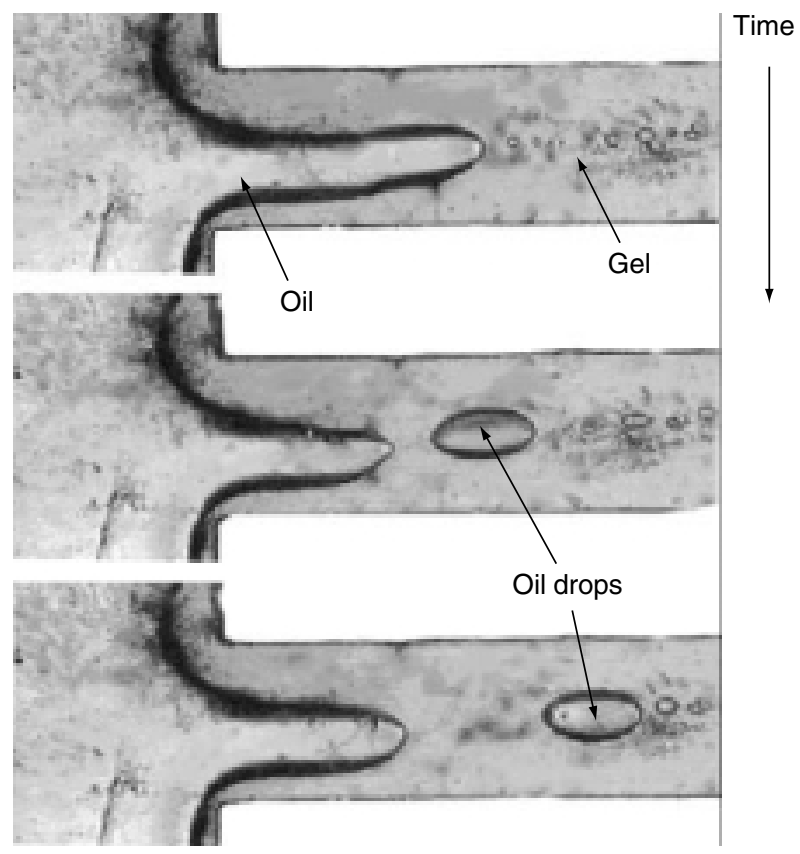

Figure 9

Oil snap-off flow near the inlet of the single capillary pore. The newly formed oil drop can flow without continuity with the injected oil.

be seen from Figure 9. After snap-off, these smaller oil droplets are able to flow by themselves, without maintaining hydraulic continuity with the oil in the inlet.

\section{DISCUSSION OF DPR MECHANISMS}

The different modes of flow taken by the oil and water can be used to explain the discrepancy between the effective permeability of the gel to oil and to water and the DPR effect. It can be shown that the permeability of a porous medium scales with the square of the mean path size, although other factors are also relevant [5]. The effective permeability of the gel to water is therefore controlled by the "pore size" of the polymer network, which is probably on the order of the polymer molecule size, $10^{-7} \mathrm{~m}$ [11]. Oil, on the other hand, creates its own, much larger, flow paths, the sizes of which were controlled by the pore diameter. In our experiments, the filament and droplet diameters were observed to be on the order of $10^{-4}-10^{-6} \mathrm{~m}$. This difference in effective path sizes gives rise to the differences in effective permeability of the two fluids.

The velocity-dependence of the permeability appears to be related to the elastic behaviour of the gel. This is supported by our observation that the changes in gel permeability were reversible as the velocity was increased or was decreased; a similar reversibility has been noted with regards to changes in temperature [14]. Also, gels are known to be very elastically deformable [15]. As the flow rate increases, some regions of the gel network expand while other regions shrink (contract), maintaining the total gel volume constant. The effective pore size would be given by the spatial distribution of these regions. As the water passes through the expanded regions, avoiding the shrunken regions, the expanded regions control the permeability. An increase in flow rate increases the size of the expanded regions, thereby increasing the permeability. This explanation supports the role of polymer elasticity in the effective permeability behaviour of the gel, as described by Equation (2).

As mentioned earlier, oil flows through the gel in the form of immiscible drops or filaments. During the formation of these drops, at constant flow rate, the pressure rises as the oil creates a narrow pathway in the centre of the pore. As soon as the snap-off occurs, the inlet pressure decreases, and the oil droplet forces its way through the gel without hydraulic continuity (Figs. 6 and 7). When an oil drop opens a path through an elastic gel, some energy is stored within the polymer gel. We hypothesise that this energy acts as the driving force for the oil droplet to flow, after snap-off. The effective permeability to oil increases with the radius and frequency of the droplets.

The dependence of permeability with the square of the path size (polymer network size for water, drop or filament size for oil) renders the gel permeability particularly sensitive to velocity. Nevertheless, the precise model for explaining this behaviour is not obvious, as the permeability correlates with velocity, and therefore with the pressure gradient, rather than with the absolute value of the pressure, which would seem more intuitive. 
Our observations allow us to dispense with several of the mechanisms that have been proposed in the past to explain the DPR effect. The "segregated pathway mechanism" [2,3] suggests that a water-based gel fills the pathways available to water, and the oil pathways remain unaffected by the gel; thus, after the gel is formed the oil will flow through the unaffected oil pathways. The water-based gel would thereby reduce the permeability to water more than that to oil. In our experiments on capillary pores and micromodels, the only pathway available for both water and oil was fully occupied by the gel. If the segregated pathway theory were valid, we would expect that the oil permeability in our capillary pore experiments would be negligible or zero. The permeability for oil was found to be 200 times greater than the water permeability for a water-wet capillary pore, indicating a large DPR. Therefore, our results show that for the gels used in our experiments, the segregated pathway mechanism is not the cause for the observed DPR effect. The swelling and shrinking mechanism [4] proposes that the gel swells in the presence of water due to the gel affinity towards the water, and the gel shrinks in the presence of oil due to gel repulsion with respect to oil. We observed no change in gel volume during water flow or oil flow. However, gel elasticity produces temporary movement when oil was forced to flow through it by creating a path for oil droplets in the centre of the channel; the gel regains its original position when the oil drop moves forward. The opening and closing actions of the polymer gel are due to the elasticity of the gel. Our observations also show that the shrinking and swelling mechanisms cannot be responsible for DPR. Another possible mechanism for DPR is the so-called wall effect [1], in which adsorbed polymer layers coat the pore walls, resulting in a pore size reduction. This layer of polymer would then restrict the flow of water near the walls, while lubricating the flow of oil. However, no such mechanism was observed during our experiments, as the crosslinked gel completely fills the pores. Thus, segregated pathways, wall effects and shrinking and swelling mechanisms were not the cause of DPR for our studies, although these mechanisms cannot be rejected, as they may occur for other systems or conditions.

\section{CONCLUSIONS}

Visual observations of flow in water-wet models have shown that oil and water flow by different flow mechanisms through an elastic polymer gel emplaced within a porous medium. Water flows through the gel network as if flowing through a porous medium, whereas the oil pushes its way through the gel, in the form of immiscible drops or filaments. This difference in effective path sizes, gel network size for water and drop/filament size for oil, gives rise to the difference in gel permeability that creates the DPR. Although segregated pathways, shrinking and swelling or wall effects were not the cause of DPR in our studies they cannot be totally rejected as possible mechanisms for other polymer gel systems because these systems may have different behaviour.

We have observed that polymer gels reduce water permeability more than oil permeability, for both capillary pores and micromodels. The extent of the permeability reduction depends on the characteristics of the gel, the flow rate, and the pore size. Both oil and water effective permeabilities follow a power-law relationship with flow velocity over a wide range of flow conditions. The velocity-dependence of the permeabilities seems to be controlled by the elasticity of polymer gels.

The observations and results presented in this paper are currently being used to provide input data, both qualitative and quantitative, for the development of mathematical models of the flow through polymer gels. This will provide a better understanding of these processes, and will help in the selection of new gel systems.

\section{ACKNOWLEDGEMENTS}

H. Al-Sharji thanks Petroleum Development Oman (PDO) for financial support and encouragement.

\section{REFERENCES}

1 Barreau, P., Lasseux, D., Bertin, H., Glénat, P. and Zaitoun, A. (1999) An Experimental and Numerical Study of Polymer Action on Relative Permeability and Capillary Pressure. Petrol. Geosci., 5, 2, 201-206.

2 White, J.L., Goddard, J.E. and Phillips, H.M. (1973) Use of Polymer to Control Water Production in Oil Wells. J. Petrol. Tech., 25, 2, 143-150.

3 Liang, J.T. and Seright, R.S. (1997) Further Investigations of Why Gels Reduce $k_{w}$ More Than $k_{0}$. SPE Prod. Fac., 12, $4,225-230$.

4 Sparlin, D.D. and Hagen, R.W. Jr. (1984) Controlling Water in Producing Operations, Part 5: Using Polyacrylamide Polymers. World Oil, 199 (July), 137-142.

5 Yang, C., Grattoni, C.A., Muggeridge, A.H. and Zimmerman, R.W. (2000) Flow of Water through ElasticallyDeformable Polymer Gels. 7th EAGE Eur. Conf. Math. Oil Recovery, Baveno, Italy, 5-8 September.

6 Yang, C. (2001) Mathematical Modelling of the Flow of Water and Oil through Polymer Gels. PhD Thesis, Imperial College, University of London.

7 Sydansk, R.D. (1990) A Newly Developed Chromium (III) Gel Technology. SPE Res. Eval., 5, 3, 346-352.

8 Dawe, R.A. and Grattoni, C.A. (1998) The Visualisation of the Pore-Scale Physics of Hydrocarbon Recovery from Reservoirs. First Break, 16, 11, 371-386.

9 Bear, J. (1988) Dynamics of Fluids in Porous Media, Dover, New York.

10 Al-Sharji, H.H. (2000) Experimental Observation and Measurement of the Flow of Water and Oil through Polymer Gels. PhD Thesis, Imperial College, University of London. 
11 Sorbie, K.S. (1991) Polymer Improved Oil Recovery, Blackie, Glasgow-London.

12 Dawe, R.A. and Zhang, Y. (1994) Mechanistic Study of Selective Action of Oil and Water Penetrating into a Gel Emplaced in a Porous Medium. J. Pet. Sci. Eng., 12, 2, 113126.

13 Couder, Y., Gérard, N., and Rabaud, M. (1986) Narrow Fingers in the Saffman-Taylor Instability. Phys. Rev. A, 34, $6,5175-5178$.
14 Tokita, M. and Tanaka, T. (1991) Reversible Decrease of Gel-Solvent Friction. Science, 253, 6, 1121-1123.

15 Östergren, K.C.E. and Trägårdh, C. (1999) Modelling and Analysis of Axial Flow through and Compression of a Non-Rigid Chromatographic Bed. Chem. Eng. J., 72, 2, 153-161.

Final manuscript received in January 2001 